\title{
2-Methoxyestradiol inhibits the proliferation and migration and reduces the radioresistance of nasopharyngeal carcinoma CNE-2 stem cells via NF-кB/HIF-1 signaling pathway inactivation and EMT reversal
}

\author{
SHUN-LONG WU ${ }^{1 *}$, YA-JUN LI ${ }^{2,3 *}$, KUI LIAO ${ }^{1}$, LEI SHI $^{4}$, NA ZHANG $^{5}$, SHUANG LIU $^{5}$, \\ YAO-YAO HU ${ }^{4}$, SHAO-LIN LI ${ }^{5}$ and YING WANG ${ }^{6}$ \\ ${ }^{1}$ Department of Oncology, The First Affiliated Hospital of Chongqing Medical University, Chongqing 400016; \\ ${ }^{2}$ Zunyi Medical College, Zunyi, Guizhou 563000; ${ }^{3}$ Department of Oncology, The Third Affiliated Hospital of \\ Zunyi Medical College, Zunyi, Guizhou 563002; ${ }^{4}$ Department of Oncology, Affiliated Hospital of Zunyi Medical College, \\ Zunyi, Guizhou 563000; ${ }^{5}$ Department of Oncology and Radiation Medicine, College of Basic Medicine, \\ Chongqing Medical University, Chongqing 400016; ${ }^{6}$ Department of Radiotherapy, \\ Chongqing Cancer Institute, Chongqing 400030, P.R. China
}

Received April 17, 2016; Accepted May 31, 2016

DOI: $10.3892 /$ or.2016.5319

\begin{abstract}
Accumulating evidence indicates that cancer stem cells (CSCs) are a source of resistance to radiation therapy (RT); however, the mechanism of this resistance remains unclear. 2-Methoxyestradiol (2-ME2) is a metabolic product of estrogen in the body. Recent studies have found that 2-ME2 regulates the activation of transcription factors, including nuclear factor

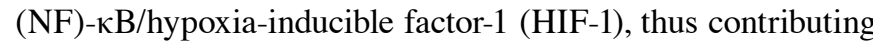
to tumor cell apoptosis and chemosensitivity. Therefore, 2-ME2 is being studied as a potential anticancer drug. The purpose of this study was to determine the effect and mechanism by which 2-ME2 inhibits nasopharyngeal carcinoma CNE-2 stem-like cell (NPCSC) proliferation and migration and reduces NPCSC radioresistance. This study has important significance for reducing the radioresistance of these cells to improve the cure rate of NPC. First, the NPCSCs were collected in a serum-free culture system and then identified by relevant experiments. The NPCSCs were treated with 2-ME2 (0-8 $\mu \mathrm{M})$ combined with X-ray exposure and Cell Counting Kit-8 (CCK-8), Transwell assay, colony formation assay, western blot analysis, RT-PCR, flow cytometry and RNA interference technology were used to explore the effect and mechanism of 2-ME2 on NPCSCs. The results showed that the microspheres collected in the serum-free
\end{abstract}

Correspondence to: Dr Ying Wang, Department of Radiotherapy, Chongqing Cancer Institute, 181 Hanyu Road, Chongqing 400030, P.R. China

E-mail: wangying_cq@hotmail.com

*Contributed equally

Key words: nasopharyngeal carcinoma, cancer stem cells, NF-кB p65, 2-methoxyestradiol, radiosensitivity culture system possessed CSC traits and radioresistance. 2-ME2 obviously inhibited NPCSC growth and migration and reduced NPCSC radioresistance. 2-ME2 decreased NF- $\kappa \mathrm{B}$ p65 and HIF-1 $\alpha$ protein expression, downregulated NF- $\kappa \mathrm{B}$ p65 nuclear localization, and reversed epithelial-mesenchymal transition (EMT). NF- $\kappa$ B p65 knockdown reduced HIF-1 $\alpha$ expression, reversed EMT, and enhanced the suppressive effect of 2-ME2 on NPCSCs. Collectively, these data indicate that 2-ME2 inhibits NPCSC proliferation and migration and reduces the radioresistance of NPCSCs via NF- $\kappa \mathrm{B} / \mathrm{HIF}-1$ signaling pathway inactivation and EMT reversal.

\section{Introduction}

Nasopharyngeal carcinoma (NPC) is a type of malignant tumor that originates in the nasopharyngeal epithelium. The primary treatment method for NPC is radiation therapy $(1,2)$. NPC is sensitive to radiation; however, according to the cancer stem cell (CSC) theory, NPC stem cells (NPCSCs) are resistant to radiation (3-5). Many factors, including nuclear factor (NF)- $\mathrm{B}$ (6) and hypoxia-inducible factor-1 (HIF-1) (7), which are overexpressed in cells under hypoxic conditions, contribute to the radioresistance of CSCs $(8,9)$.

$\mathrm{NF}-\kappa \mathrm{B}$, a nuclear transcription factor, is overexpressed in various cancers containing NPCs, and p65 is a key subunit required for functional $N F-\kappa B$ activity $(10,11)$. Previous studies have found that $N F-\kappa B$ regulates epithelial-mesenchymal transition (EMT), thus contributing to cancer occurrence and progression (12). Decreased protein expression of the epithelium marker E-cadherin (E-cad) and increased protein expression of $\mathrm{N}$-cadherin $(\mathrm{N}$-cad) and vimentin are direct evidence of the occurrence of EMT (13). 2-Methoxyestradiol (2-ME2), an endogenous estradiol metabolite, is being investigated in clinical trials as an anticancer agent (14). 2-ME2 regulates $\mathrm{NF}-\kappa \mathrm{B}$ activation to influence apoptosis and chemoresistance 
in various tumor cell lines, including leukemia K562 cells (15), medulloblastoma cells (16), prostate cancer cells (17) and pancreatic cancer cells (18). Thus, we speculated that 2-ME2 may play a vital role in the proliferation, migration and radioresistance of NPCSCs via the regulation of NF- $\kappa$ B p65 activity.

Solid tumors generally have a hypoxic microenvironment (19). HIF-1 $\alpha$, a unique subunit of HIF-1, which functions in the cellular response to oxygen levels and which is overexpressed in solid tumors, is closely associated with CSC resistance to treatment (20-22). A previous study found that 2-ME2 decreased the HIF-1 $\alpha$-binding activity, affected the expression of downstream genes, inhibited tumor growth and angiogenesis and augments paclitaxel efficacy in head and neck squamous cell carcinoma (23). However, whether 2-ME2 affects NPCSC proliferation, migration and treatment resistance through the regulation of HIF-1 $\alpha$ remains unclear.

The objective of this study was to determine the antitumor effects of 2-ME2 on NPCSCs and to demonstrate the underlying mechanism involved in the antitumor effects of 2-ME2 through the suppression of the NF- $\mathrm{KB} / \mathrm{HIF}-1$ signaling pathway. 2-ME2 could potentially become a novel radiotherapy sensitization agent for clinical use.

\section{Materials and methods}

Reagents. The human NPC cell line CNE-2 was obtained from the Tumor Laboratory of Chongqing Medical University (Chongqing, China). Penicillin-streptomycin (1\%), fetal bovine serum (FBS), RPMI-1640 culture medium and Dulbecco's modified Eagle's medium/F12 (DMEM/F12; 1:1 volume) were purchased from Gibco (Grand Island, NY, USA). B27 supplement (2\%) was purchased from Invitrogen (Carlsbad, CA, USA). Accutase was purchased from Innovative Cell Technologies, Inc. (San Diego, CA, USA). Human recombinant basic fibroblast growth factor (bFGF) and epidermal growth factor (EGF) were purchased from Peprotech, Inc. (Rocky Hill, NJ, USA). 2-ME2 was purchased from Selleck Chemicals (Shanghai, China). Real-time polymerase chain reaction (RT-PCR) kits were purchased from Takara Biotechnology Co., Ltd. (Dalian, China). Transwell chambers with an $8-\mu \mathrm{m}$ aperture were purchased from Beijing Mingyangkehua Biotechnology Co., Ltd. (Beijing, China). Anti-CD133-PE antibody for flow cytometry (FCM) was purchased from Miltenyi Biotec (Beijing, China). The lentiviral vector encoding NF- $\mathrm{B}$ p65-shRNA (pMAGic 7.1) was obtained from Shanghai Sunbio Co. (Shanghai, China). Mouse antihuman primary antibodies directed against NF- $\kappa \mathrm{B}$ p65, lamin B1, vimentin, E-cad, and $\mathrm{N}$-cad were purchased from Cell Signaling Technology, Inc. (Beverly, MA, USA). The mouse anti-human primary antibodies directed against HIF- $1 \alpha$ and GAPDH and secondary goat anti-mouse IgG antibody were purchased from Wuhan Boster Biological Engineering Co., Ltd. (Wuhan, China). A modified BCA protein assay kit, an allergic ECL chemiluminescence reagent kit and an SDS-PAGE gel preparation kit were purchased from Beyotime Biotech (Jiangsu, China). Cell proliferation-toxicity testing kits [Cell Counting Kit-8 (CCK-8)] were purchased from Dojindo Laboratories Co., Ltd. (Kumamoto, Japan). An inverted fluorescence microscope was obtained from Nikon Corporation (Tokyo, Japan), an upright fluorescence microscope was obtained from Olympus Corp. (Tokyo, Japan), a flow cytometer was obtained from Bio-Rad Laboratories, Inc. (Hercules, CA, USA), and a linear accelerator for radiotherapy was obtained from Varian Medical Systems, Inc. (Palo Alto, CA, USA).

Cell culture. To culture the parental CNE-2 cell lines, CNE-2 cells were cultured in RPMI-1640 supplemented with $1 \%$ penicillin-streptomycin and $10 \% \mathrm{FBS}$, placed in an incubator with $5 \% \mathrm{CO}_{2}$ at $37^{\circ} \mathrm{C}$, and passaged every other day.

To enrich stem-like cells, a non-adhesive culture system of serum-free medium (SFM) consisting of DMEM/F12 supplemented with $20 \mathrm{ng} / \mathrm{ml} \mathrm{bFGF}, 20 \mathrm{ng} / \mathrm{ml}$ EGF, 2\% B27 supplement and $1 \%$ penicillin-streptomycin was used to collect NPCSCs. The parental CNE-2 cells were re-suspended in T25, placed vertically in an incubator, shaken several times a day, and passaged every 4-6 days until the cells formed microspheres.

To culture the NPCSCs, the microsphere suspension was cultured in SFM and passaged every 4-6 days. When the cells were passaged, the tumor spheroids were dissociated with Accutase into single cells, transferred to new T25 containing new SFM, and placed in an incubator with $5 \% \mathrm{CO}_{2}$ at $37^{\circ} \mathrm{C}$.

NPCSC re-differentiation assay. NPCSCs were collected, dissociated into single cells with Accutase, seeded in a 6-well plate with RPMI-1640 containing $10 \%$ FBS, and placed in an incubator with $5 \% \mathrm{CO}_{2}$ at $37^{\circ} \mathrm{C}$. After 3-5 days, the changes in cellular morphology were observed using an inverted microscope.

Transwell migration assay. The parental CNE-2 cells and the NPCSCs were dissociated into single cells that were then re-suspended $\left(2 \times 10^{5}\right.$ cells $\left./ \mathrm{ml}\right)$ in SFM. The upper chamber was loaded with a 100- $\mu 1$ cell suspension, and the lower chamber was loaded with a $500 \mu 110 \%$ serum-containing medium. The cells were fixed after $24 \mathrm{~h}$ with methanol and stained with crystal violet. Five random fields were analyzed, and the number of cells invading through the membrane was counted under a microscope (Olympus Corp.). Three independent experiments were performed.

Soft agar colony assay. The parental cells and the NPCSCs were dissociated into single cells and then re-suspended $\left(10^{3}\right.$ cells $\left./ \mathrm{ml}\right)$ with SFM in $0.3 \%$ agar overlaid in 6-well plates ( $2 \mathrm{ml} /$ well) containing a $0.6 \%$ agar base. After the cells were incubated for 3 weeks, the spheroids $>0.2 \mathrm{~mm}$ in diameter were evaluated using fluorescence microscopy. Three independent experiments were performed.

$R T$-PCR. To quantify gene expression, RT-PCR was performed with SYBR-Green. In brief, an RNAiso reagent was used to extract total RNA, and a reverse transcriptase kit was used to synthesize the cDNA according to the manufacturer's instructions. Then, PCR was performed. The expression levels of target genes relative to GAPDH were determined using a SYBR-Green-based comparative CT method (relative fold-change, $\left.2^{-\Delta \Delta C t}\right)$. The primers were designed by Sangon Biotech Co., Ltd. (Shanghai, China), and are as follows: NF-кB p65 forward, 5'-GGAGCACAGATACCACCAAGA-3' and 
reverse, 5'-CGGCAGTCCTTTCCTACAAG-3'; HIF-1 $\alpha$ forward, 5'-CTGCCAACCCCGAAATGACAT-3' and reverse, 5'-CGCCGCTTAATAGCCCTCTG-3'; Bmi-1 forward, 5'-CCTGATGTGTGTGCTTTGTG-3' and reverse, 5'-GGTC TGGTCTTGTGAACTTGG-3'; Twist1 forward, 5'-GAGCAA GATTCAGACCCTCAA-3' and reverse, 5'-CATCCTCCAGA CCGAGAAG-3'; Oct4 forward, 5'-AGCCCTCATTTCACCAG GCC-3' and reverse, 5'-TGGGACTCCTCCGGGTTTTG-3'; GAPDH forward, 5'-ACGGGAAGCTCACTGGCATGG-3' and reverse, 5'-GGTCCACCACCCTGTTGCTGTA-3'.

The NPCSCs are treated with 2-ME2 and grouped. The NPCSCs were dispersed into single cells, suspended in pre-made 4,8 and $0 \mu \mathrm{M}$ (control) 2-ME2 solution and then placed in an incubator with $5 \% \mathrm{CO}_{2}$ at $37^{\circ} \mathrm{C}$ for $24 \mathrm{~h}$.

Colony formation assay. Cells that had been treated with 2-ME2 for $24 \mathrm{~h}$ or that were in the early log phase were dispersed, plated in 6-well plates at 200, 400, 800 and 2,000 cells/well and then treated with $0,2,4$ and 8 Gy irradiation. The cells were incubated for 12 days to allow colony formation. Subsequently, the cells were fixed and stained with $0.5 \%$ crystal violet, and the number of colonies containing $>50$ cells was counted. Survival curves were fitted using the multi-target click model in GraphPad Prism 5.0 software. Each point on the survival curve represents the mean surviving fraction (SF) from at least three independent experiments.

Flow cytometric assay. The expression of $\mathrm{CD}_{133^{+}}$, a surface marker of CSCs, was analyzed using FCM. The cells were dissociated into single cells, washed and re-suspended in PBS. Then, the cells were counted, adjusted to $1 \times 10^{6}$ cells/group, and incubated with the appropriate concentration of anti-CD133-PE (Miltenyi Biotec). The proportion of $\mathrm{CD}_{133^{+}}$cells in each sample was detected by FCM.

Western blot analysis. Cytoplasmic, nuclear or total protein extraction and sample preparation for western blot analysis were performed as previously described (24). The membranes were then incubated with one or more of the following primary antibodies: anti-NF- $\mathrm{KB}$ p65, anti-lamin B1, anti-E-cad, anti-N-cad, and anti-vimentin (all 1:1,000), as well as anti-GAPDH and anti-HIF-1 $\alpha$ (each 1:200). The proteins of interest were then detected with goat anti-mouse secondary antibody $(1: 3,000)$. The band intensities were detected using a western blot analysis system. The proteins were normalized to GAPDH or lamin B1 and quantified using the ChemiDoc ${ }^{\mathrm{TM}} \mathrm{XRS}$. Three independent experiments were performed.

Cell viability test. Cells that had been treated with 2-ME2 $(0-8 \mu \mathrm{M})$ for $24 \mathrm{~h}$ were dispersed into single cells, counted, and then seeded into 96 -well plates at $2 \times 10^{4}$ cells/well. Each group was set in 8 repeated wells and each well was loaded with $20 \mu \mathrm{l}$ of CCK-8. After the plates were placed in an incubator for $3 \mathrm{~h}$, cell viability was detected using ELISA.

Immunofluorescence staining. The cells that had been treated with 2-ME2 $(0-8 \mu \mathrm{M})$ for $24 \mathrm{~h}$ were plated onto poly-L-lysine-coated glass coverslips, fixed in $4 \%$ paraformaldehyde for $20 \mathrm{~min}$ at room temperature, permeabilized with $0.1 \%$ Triton X-100 and blocked with $10 \%$ BSA. The slides were incubated overnight at $4{ }^{\circ} \mathrm{C}$ with mouse anti-NF- $\kappa \mathrm{B}$ p 65 followed by incubation with secondary fluorescein-conjugated antibodies [goat anti-mouse-IgG, tetraethyl rhodamine isothiocyanate (TRITC)] at room temperature for $1 \mathrm{~h}$. The slides were counterstained with 4'-6-diamidino-2-phenylindole (DAPI) and analyzed under a fluorescence microscope. Three independent experiments were performed.

p65-shRNA-infected cells. Gene knockdown targeting NF-кB p65 was performed as previously described (24). Two oligonucleotides were used to target NF- $\mathrm{KB}$ p65. The cells were grown to the log phase in T25, dispersed into single cells and transfected with the virus shRNA or virus non-carrier in SFM. The levels of NF- $\mathrm{kB}$ p65 gene silencing and protein expression were evaluated by RT-PCR and western blot analysis, respectively.

Statistical analysis. The values are expressed as the means \pm standard deviation (SD). Differences between groups were analyzed by Student's t-tests. Differences among three groups were analyzed using one-way ANOVA, and differences between any two groups were analyzed using least significant difference (LSD) tests. All statistical analyses were performed using SPSS version 16.0 for Windows. Values of ${ }^{*} p<0.05$ were considered significant, and values of ${ }^{* *} \mathrm{p}<0.01$ were considered very significant.

\section{Results}

NPCSCs which were derived from CNE-2 cells acquired stem-like characteristics and radioresistance. The parental CNE-2 cells were cultured and routinely passaged in RPMI-1640 medium containing 10\% FBS (Fig. 1A left, upper panel). Cells formed microspheres in the SFM approximately 8-10 days after being suspended (Fig. 1A right, upper panel). The microspheres maintained a strong ability to grow after repeated passages (Fig. 1A left, panel below) and differentiated into spindle adherent cells when re-cultured in medium containing 10\% FBS (Fig. 1A right, panel below). These data demonstrated that the spherical cells have strong self-renewal and differentiation abilities. These abilities were subsequently identified as stem cell characteristics of NPCSCs. To identify the stem cell characteristics of the spherical cells, soft agar cloning and Transwell assays were used to detect the selfrenewal and migration abilities, respectively, of these cells in vitro. The number of CNE-2 colonies was significantly reduced compared with the number of spherical cells (Fig. 1B; $\mathrm{p}<0.01$ ). The number of CNE-2 cells that migrated to the other side of the membrane was significantly decreased compared with the number of spherical cells that migrated (Fig. 1C; $\mathrm{p}<0.01$ ). The cell surface antigen $\mathrm{CD} 133^{+}$was used as a CSC-specific marker, and the proportion of $\mathrm{CD} 133^{+}$cells was detected by FCM. The results revealed that the proportion of $\mathrm{CD}_{133^{+}}$cells was $85.2 \pm 5.88 \%$ for spherical cells and $1.47 \pm 0.45 \%$ for CNE-2 cells ( $<<0.01$ ) (Fig. 1D). Several genes, including Bmi-1, Twist1, and Oct4, which are associated with CSC properties, were quantitatively analyzed by RT-PCR. We found that the expression levels of these genes were significantly 
A

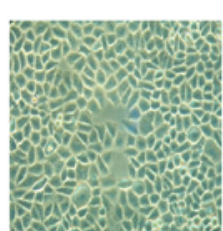

CNE-2

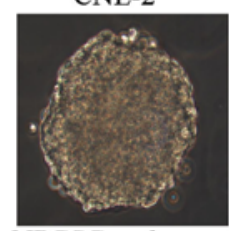

NPCSC undergone several passages

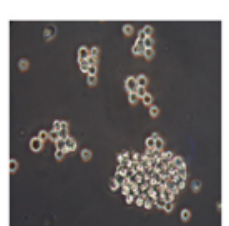

NPCSC formation

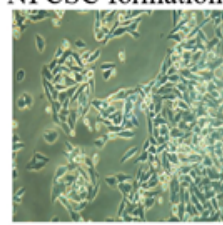

NPCSC

redifferentiation
B

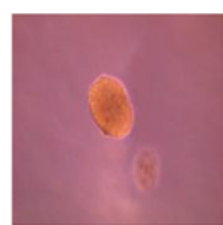

NPCSCs

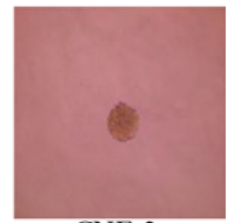

CNE-2

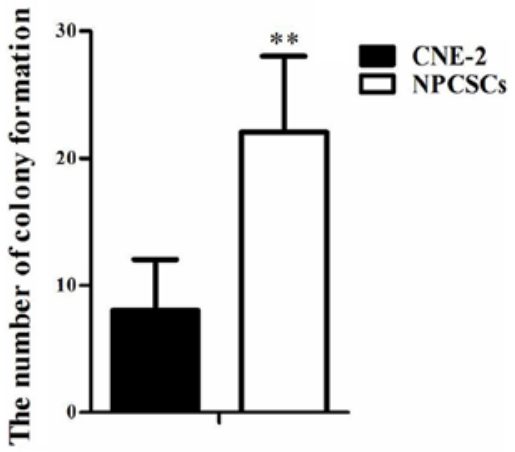

C

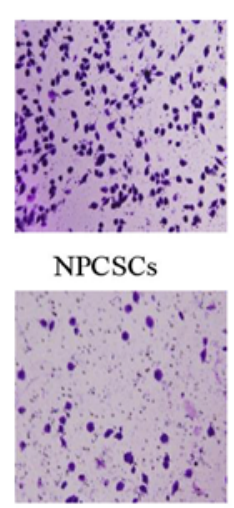

CNE-2

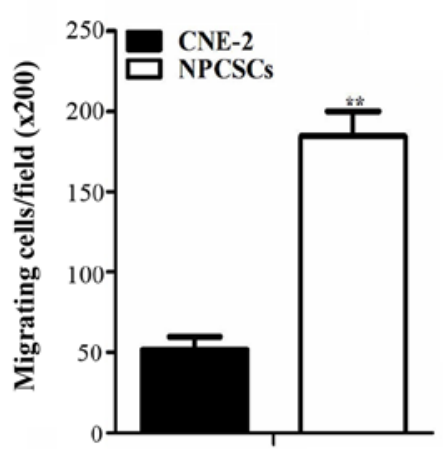

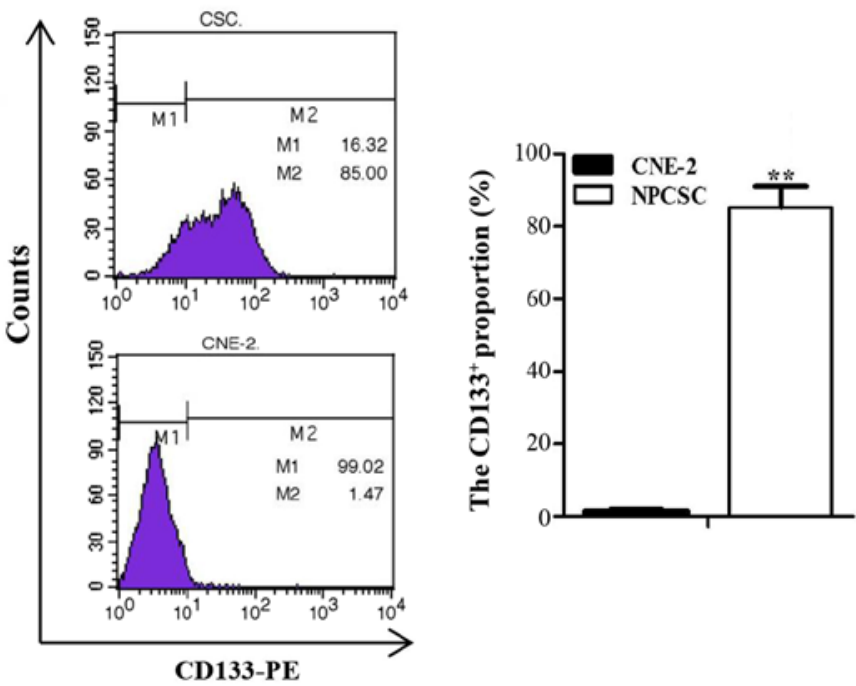

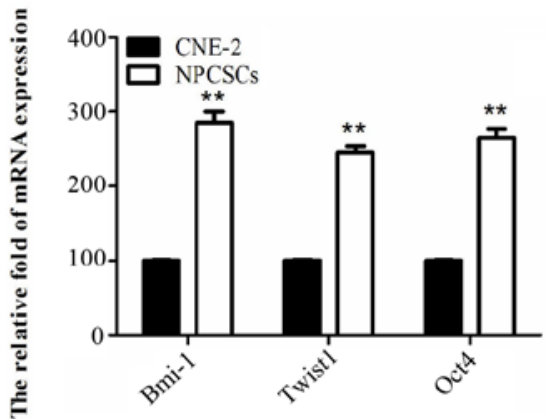

F

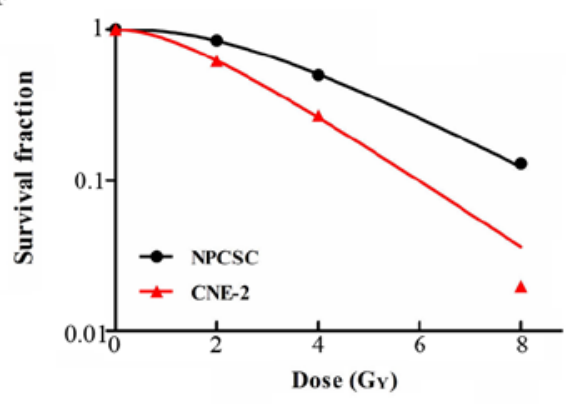

Figure 1. The NPCSCs are enriched from CNE-2 cells in a serum-free culture system, and stemness is analyzed using cancer stem cell (CSC)-related assays. (A) The NPCSCs were enriched from CNE-2 cells in a serum-free culture system, passaged and re-differentiated in a serum-containing media. (B) Comparison of colony formation between NPCSCs and parental CNE-2 cells using a soft agar colony assay. (C) Comparison of the migratory abilities of NPCSCs and parental CNE-2 cells by Transwell assay. (D) The proportion of CD133 ${ }^{+}$cells was detected by flow cytometry (FCM). (E) The relative RNA expression levels of the stemness-related genes Bmi-1, Twist1, and Oct4 were detected by RT-PCR. (F) Colony formation assay was used to detect the radiosensitivity of NPCSCs and CNE-2 cells. The survival curve of the surviving fraction was generated using GraphPad software with a multi-target single-hit model. All representative micrographs were taken at $\mathrm{x} 200$ magnification. The data are expressed as the means $\pm \mathrm{SD}$ of three independent experiments. ${ }^{* *}$ p $<0.01$ compared with the parental CNE-2 cells. NPCSCs, nasopharyngeal carcinoma stem-like cells.

increased in spherical cells compared with those in the CNE-2 cells (Fig. 1E). Colony formation assay was used to investigate the radiosensitivity of the spherical cells. The survival curve of the SF was generated using GraphPad software with a multi-target single-hit model. This result demonstrated that the spherical cells were more resistant to radiation therapy compared with the CNE-2 cells (Fig. 1F). We concluded that the spherical cells presented strong stemness, and we used these cells in subsequent experiments as NPCSCs.

2-ME2 inhibits NPCSC proliferation and migration and reduces NPCSC radioresistance. The compact and spherical 
A

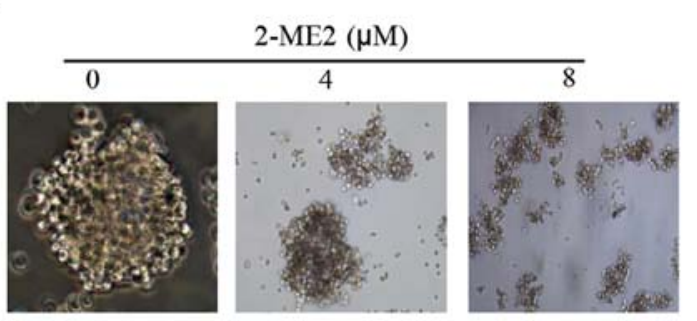

C

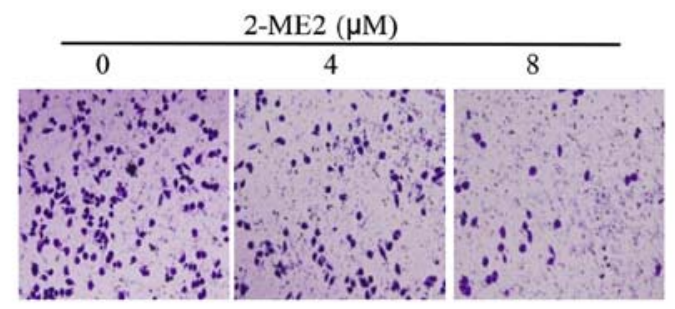

B
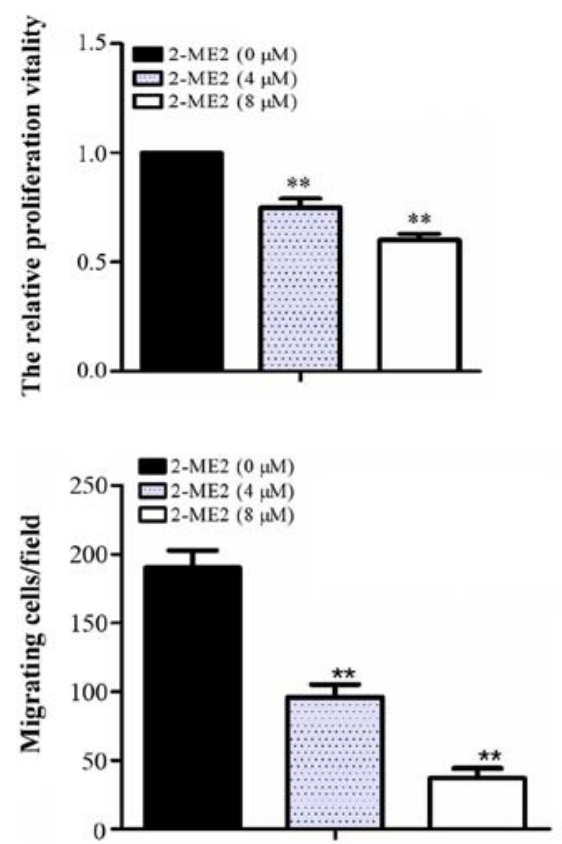

D

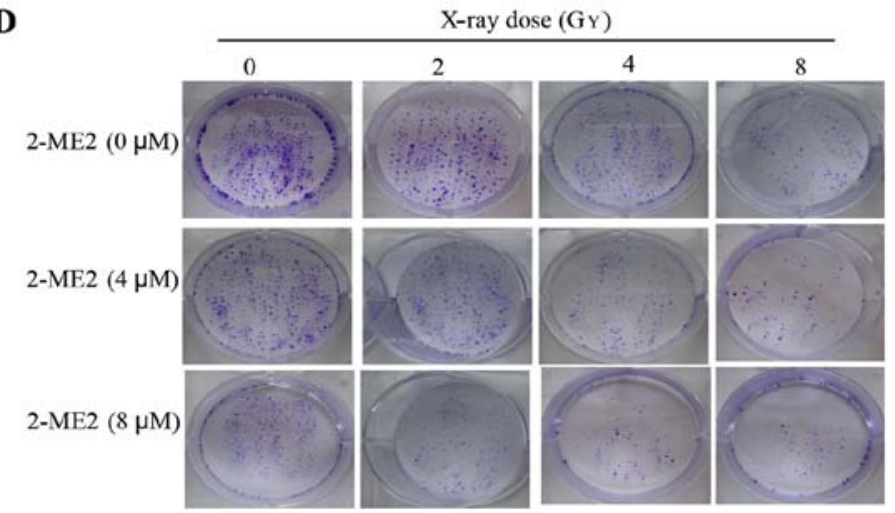

E

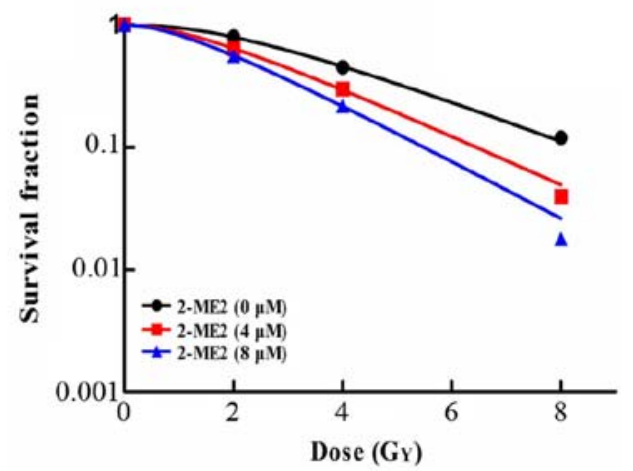

Figure 2. 2-Methoxyestradiol (2-ME2) inhibits NPCSC proliferation and migration and reduces NPCSC radioresistance. (A) NPCSCs were seeded in suspension and treated with various concentrations of 2-ME2 $(0-8 \mu \mathrm{M})$ for $24 \mathrm{~h}$. Images of the NPCSCs were captured using inverted microscopy. (B) After the NPCSCs were seeded in suspension and treated with 2-ME2 (0-8 $\mu \mathrm{M})$ for $24 \mathrm{~h}$, the cells were collected and dissociated with Accutase. A Cell Counting Kit-8 (CCK-8) assay was used to measure cell viability. (C) After the NPCSCs were seeded in suspension and treated with $2-\mathrm{ME} 2(0-8 \mu \mathrm{M})$ for $24 \mathrm{~h}$, a Transwell assay was used to measure the migratory ability of the NPCSCs (magnification, $\mathrm{x} 200$ ). The quantification of the migrated cells is shown on the right. (D) To investigate the effect of 2-ME2 on the radiosensitivity of NPCSCs, a colony formation assay was used. Cells were treated with 2-ME2 for $24 \mathrm{~h}$ followed by 2-8 Gy irradiation and allowed to grow for 12 days. (D) Representative images of colony formation are shown. (E) Survival fractions were obtained from the results of the colony forming assays. Survival curves were fitted using the multi-target click model. 2-ME2 enhanced the radiosensitivity of NPCSCs, with SERs of 1.20 for the $4 \mu \mathrm{M}$ 2-ME2 group and 1.39 for the $8 \mu \mathrm{M} 2$-ME2 group. All data are presented as the means \pm SD from three independent experiments. ${ }^{* *} \mathrm{p}<0.01$ compared with controls treated with $0 \mu \mathrm{M}$ 2-ME2 (DMSO replaced). NPCSCs, nasopharyngeal carcinoma stem-like cells.

growth of NPCSCs that had been treated with 2-ME2 for $24 \mathrm{~h}$ dispersed gradually with increasing concentrations of 2-ME2 as observed under an inverted microscope (Fig. 2A). A CCK-8 assay was used to detect cell proliferation. Cell proliferation clearly decreased with increasing concentrations of 2-ME2 (Fig. 2B). A Transwell assay was used to detect the migratory abilities of these cells. The numbers of cells crossing the membrane were $190 \pm 12,96 \pm 7$, and $37 \pm 5$ (Fig. 2C) for the groups treated with 2-ME2 (0, 4 and $8 \mu \mathrm{M}$, respectively), which indicated that 2-ME2 inhibited NPCSC migration. To determine the radiosensitivity effect of 2-ME2 on the NPCSCs, a colony formation assay was performed (Fig. 2D). As depicted in Fig. 2E, the shoulder area of the survival curves significantly narrowed, and the SFs at each dose (2, 4 and $8 \mathrm{~Gy}$ ) decreased in a 2-ME2 concentration-dependent manner. The SER of the 4 and $8 \mu \mathrm{M}$ groups was $1.20(\mathrm{p}<0.05)$ and $1.39(\mathrm{p}<0.05)$, respectively, indicating that 2-ME2 reduced NPCSC radioresistance.

2-ME2 decreases the proportion of $\mathrm{CD} 133^{+}$cells, inhibits the $N F-\kappa B$ p65/HIF-1 $\alpha$ signaling pathway, and reverses EMT in NPCSCs. To determine the molecular mechanism of 2-ME2 inhibition of NPCSC growth and migration and the reduction of NPCSC radioresistance, FCM, cellular immunofluorescence and western blot analyses were performed. The FCM results indicated that the proportion of $\mathrm{CD}_{133^{+}}$ cells decreased significantly in the groups treated with higher concentrations of 2-ME2 (Fig. 3A), thus demonstrating that 2-ME2 may decrease NPCSC stemness. According to cellular immunofluorescence, the location of NF- $\mathrm{kB}$ p65 in 
A

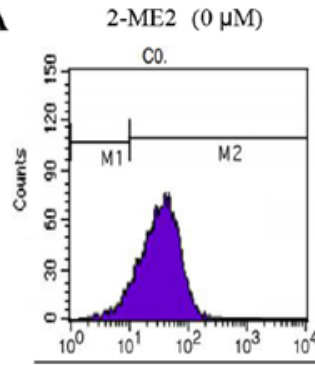

2-ME2 $(4 \mu \mathrm{M})$

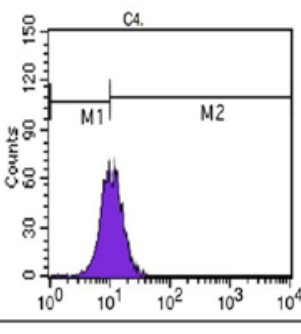

2-ME2 $(8 \mu \mathrm{M})$

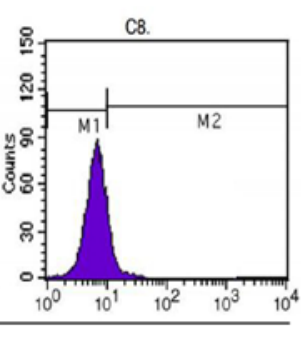

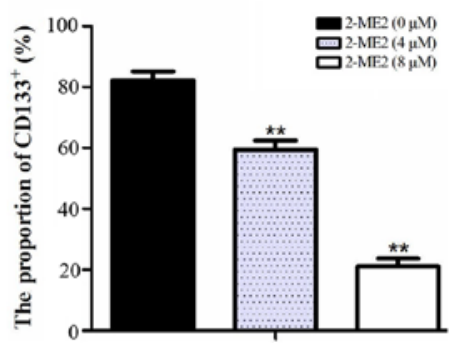

B

DAPI

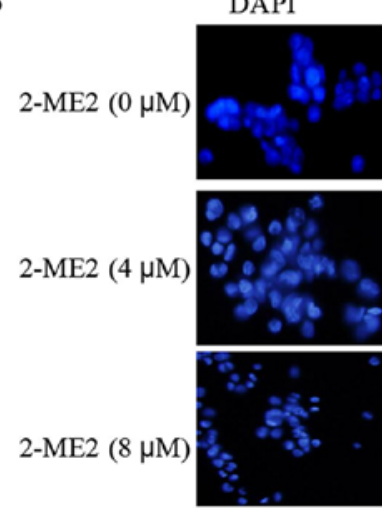

NF- KB p 65

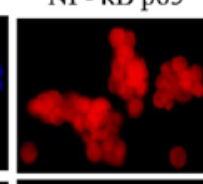

Merge
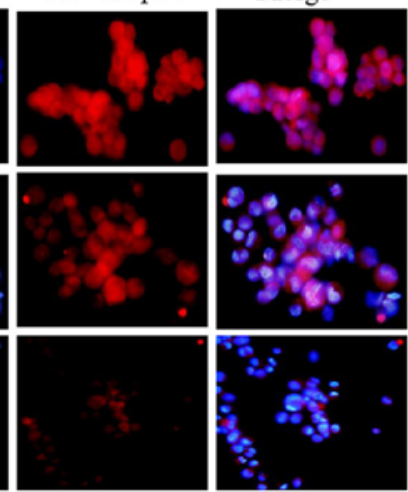

C
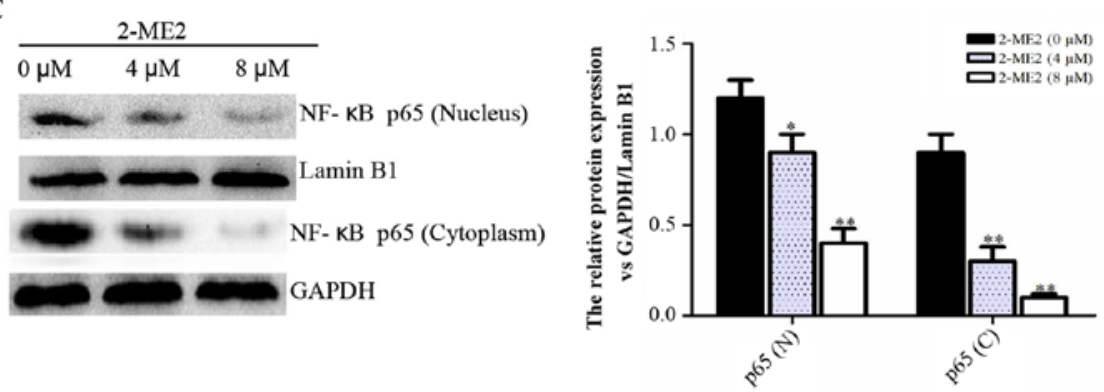

D
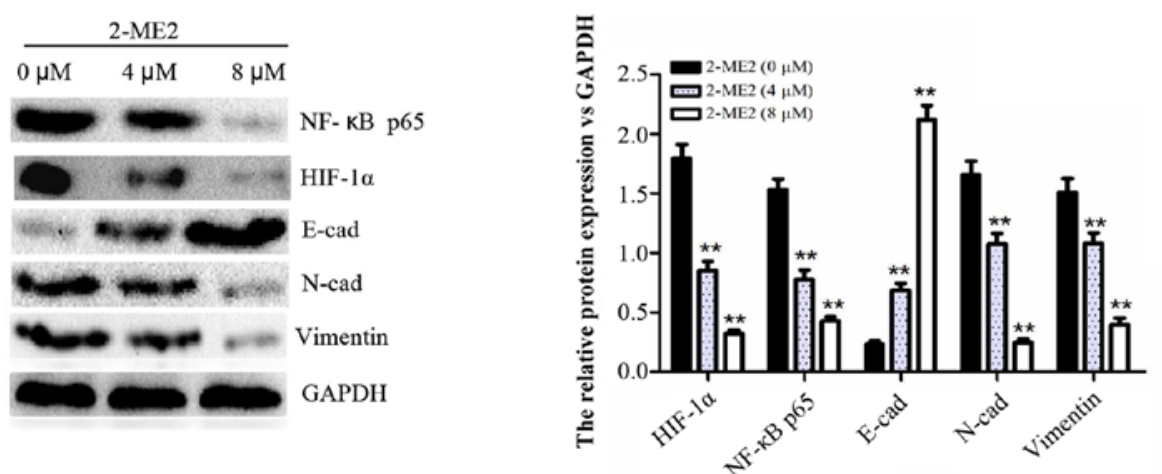

Figure 3. Effect of 2-ME2 on NPCSCs at the molecular level. (A) Flow cytometry (FCM) results showed that the proportion of CD133 ${ }^{+}$cells in NPCSCs treated with 4 or $8 \mu \mathrm{M}$ 2-methoxyestradiol (2-ME2) was reduced significantly, thus indicating that 2-ME2 attenuated the stemness traits of these NPCSCs.

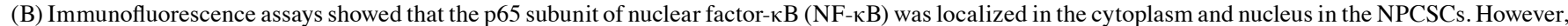
the nuclear accumulation of NF- $\mathrm{BB}$ p 65 was blocked significantly in a dose-dependent manner when NPCSCs were treated with 2-ME2 (0-8 $\mu \mathrm{M})$ for $24 \mathrm{~h}$. (C) Western blot analysis showed that nuclear and cytoplasmic p65 levels were decreased in a dose-dependent manner by 2 -ME2 treatment for $24 \mathrm{~h}$ (left panel). The changes in protein expression were analyzed by densitometric quantification (right panel). Lamin B1 was used as a nuclear loading control. (D) Western blot analyses revealed that the protein expression of NF- $\mathrm{kB}$ p65, hypoxia-inducible factor-1 $\alpha$ (HIF-1 $\alpha$ ) and mesenchymal markers in NPCSCs treated with 2-ME2 for $24 \mathrm{~h}$ was effectively reduced in a dose-dependent manner, whereas the protein expression of the epithelial marker E-cadherin was increased (left panel). The changes in protein expression were analyzed by densitometric quantification (right panel). The results revealed that 2-ME2 inhibited the protein expression of NF-KB p65 and HIF-1 $\alpha$ and reversed epithelial-mesenchymal transition (EMT). Objective magnification, $\mathrm{x} 400$. The data are expressed as the means \pm SD of three independent experiments. ${ }^{*} p<0.05 ;{ }^{* *} p<0.01$ compared with the respective controls. NPCSCs, nasopharyngeal carcinoma stem-like cells.

the nucleus is representative of significantly decreased activation (Fig. 3B). Furthermore, western blot analysis revealed that the protein expression of $\mathrm{NF}-\kappa \mathrm{B}$ p65 in the nucleus and cytoplasm and the protein expression of total HIF-1 $\alpha$ 
A

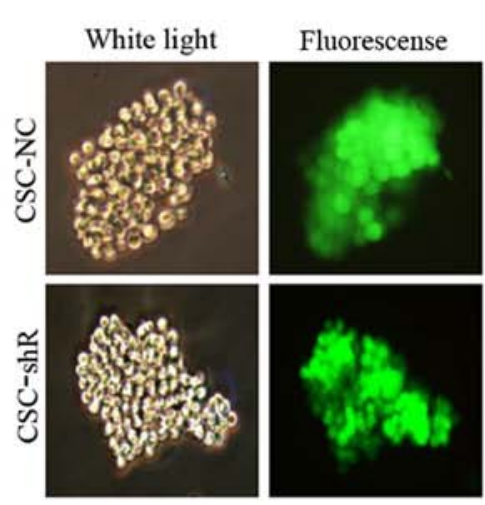

B

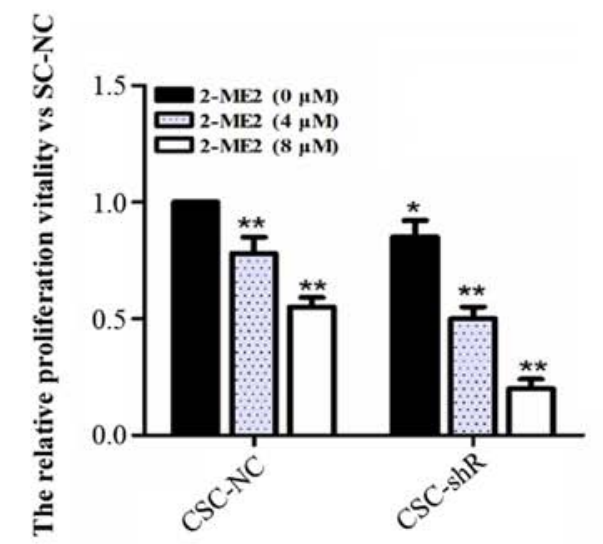

C
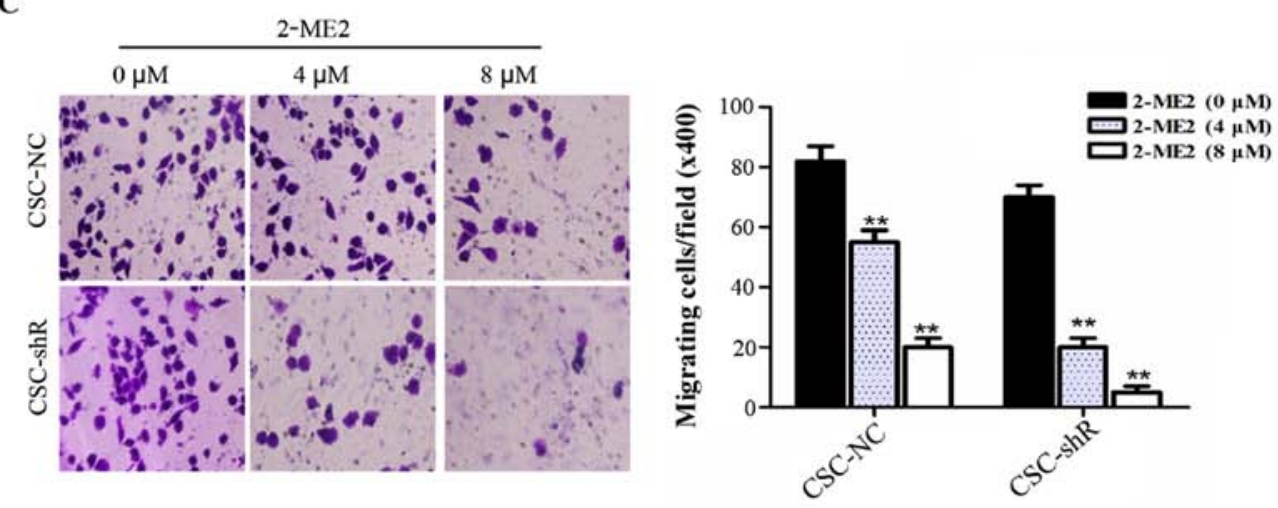

D

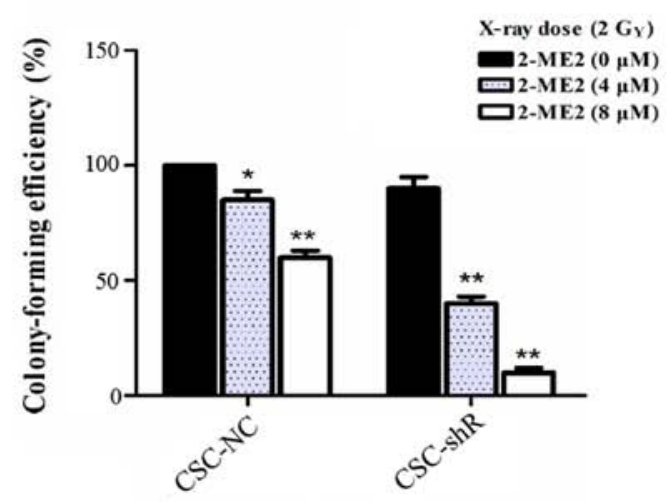

Figure 4. Nuclear factor- $\mathrm{kB}(\mathrm{NF}-\mathrm{\kappa B}) \mathrm{p} 65$ knockdown enhances the effect of 2-methoxyestradiol (2-ME2) on NPCSCs. (A) The negative control cancer stem cell (CSC)-NC (upper panel) and transfected control CSC-shR (lower panel) were observed under white light (left panel) and fluorescence (right panel) microscopy after $72 \mathrm{~h}$ (magnification, x200). (B) A CCK-8 assay demonstrated that NF-kB p65 knockdown enhanced the 2-ME2-induced inhibition of NPCSC proliferation. (C) Representative micrographs (left panel; magnification, $\mathrm{x} 400$ ) showing the migration of CSC-NC and CSC-shR after these cells were treated with 2-ME2 (0-8 $\mu \mathrm{M})$; the quantification of the migrated cells is shown on the right. (D) A colony formation assay was used to measure the effect of NF- $\kappa \mathrm{B}$ p65 on the radiosensitivity of NPCSCs treated with 2-ME2 $(0-8 \mu \mathrm{M})$ after exposure to 2 Gy radiation. The data demonstrated that 2-ME2 inhibited NPCSC colony formation after radiation exposure in a dose-dependent manner; NF- $\mathrm{kB}$ p65 knockdown further enhanced the anti-radioresistance effects of 2-ME2 on NPCSCs. The data are expressed as the means \pm SD of three independent experiments. "p $<0.05 ;{ }^{* *} p<0.01$ compared with the respective negative controls. NPCSCs, nasopharyngeal carcinoma stem-like cells.

were reduced (Fig. 3C and D), which indicated that the $\mathrm{NF}-\kappa \mathrm{B} / \mathrm{HIF}-1$ signaling pathway was inhibited. Additionally, the expression of the EMT marker E-cad increased, whereas the expression of $\mathrm{N}$-cad and vimentin decreased (Fig. 3D), which indicated reversal of EMT. Based on these results, we concluded that 2-ME2 decreased the cell stemness, inhibited the NF- $\mathrm{N} / \mathrm{HIF}-1$ signaling pathway, and reversed EMT in NPCSCs.
$N F-\kappa B$ p65 knockdown enhances the effect of 2-ME2 on NPCSCs. Lentiviral infection was performed in NPCSCs using a virus-shRNA-NF- $\mathrm{B}$ p65 solution and stably transfected cell lines. NPCSCs transfected with the lentivirus-mediated RNA targeting NF- $\kappa \mathrm{B}$ p65 (CSC-shR) and with the empty retroviral vector (CSC-NC) were also established (Fig. 4A). To investigate the role of NF- $\kappa \mathrm{B}$ p65 in the effect of 2-ME2 on NPCSCs, CCK-8, Transwell, and colony formation assays 

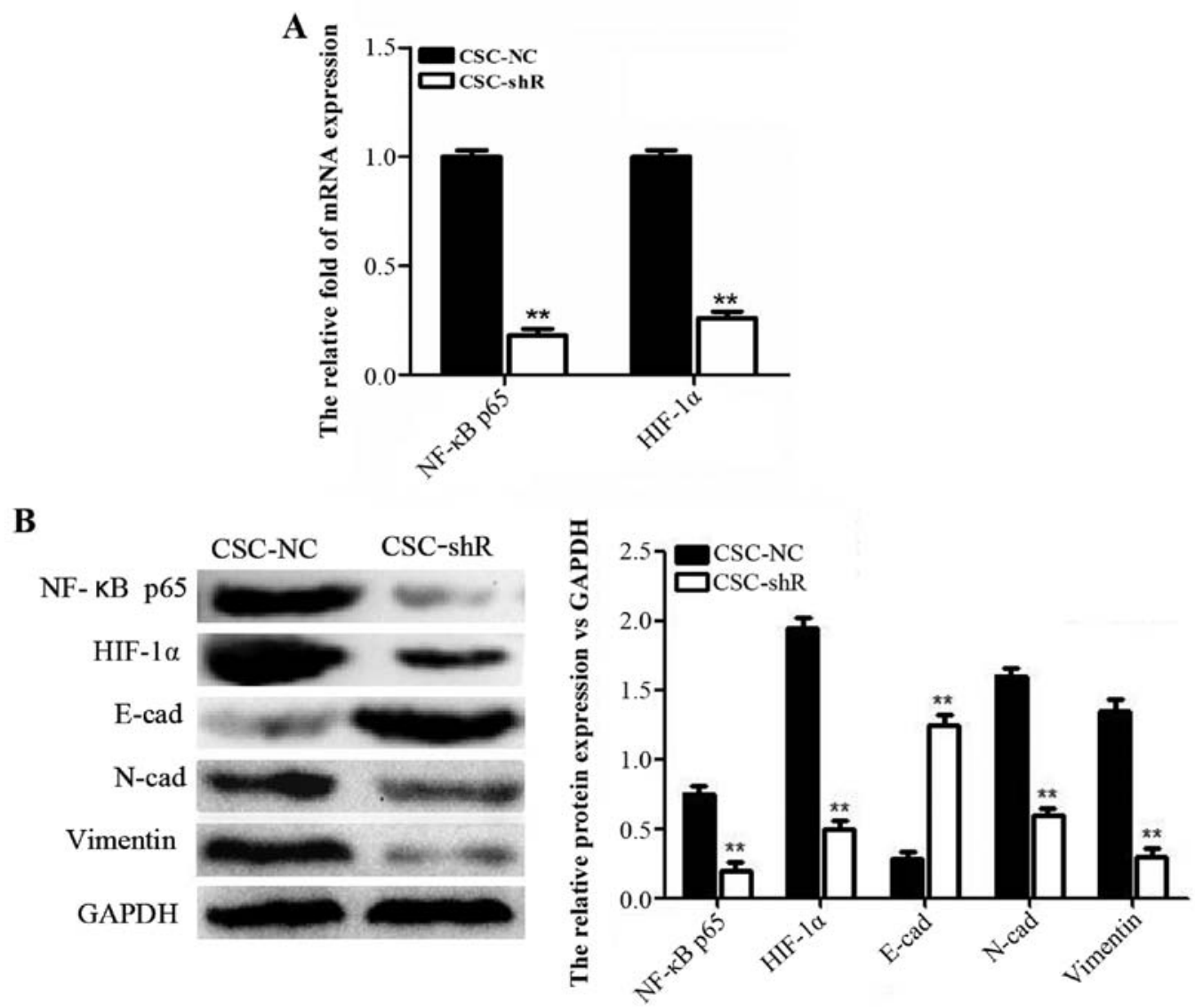

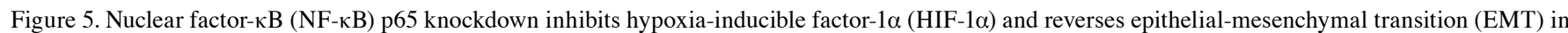
NPCSCs. (A) RT-PCR was used to quantify NF-kB p65 and HIF-1 $\alpha$ mRNA expression, and the results showed that NF-kB p65 and HIF-1 $\alpha$ mRNA expression decreased significantly after silencing of NF- $\kappa B$ p65. (B) Western blot analysis showed that the protein expression of NF- $\kappa B$ p 65 and HIF-1 $\alpha$ and mesenchymal markers in the CSC-shR cells were effectively decreased compared with levels in the CSC-NC cells, whereas the protein expression of the epithelial marker E-cadherin was increased (left panel). The differences in protein expression were analyzed by densitometric quantification (right panel). The data are expressed as the means \pm SD of three independent experiments. ${ }^{* *} \mathrm{p}<0.01$ compared with the CSC-NC cells. NPCSCs, nasopharyngeal carcinoma stem-like cells.

were performed. The viability of the CSC-NC cells was significantly decreased in a 2-ME2 concentration-dependent manner. Additionally, NF- $\mathrm{kB}$ p 65 knockdown enhanced the effect of 2-ME2 on NPCSCs (Fig. 4B). A Transwell assay was used to evaluate the migration of NF- $\mathrm{kB}$ p65-silenced NPCSCs in vitro. The number of CSC-shR cells that migrated to the other side of the membrane decreased more significantly with increasing concentrations of 2-ME2 compared to the CSC-NC (Fig. 4C). These results indicated that silencing of NF- $\kappa B$ p65 enhanced the effect of 2-ME2 on the proliferation and migration of NPCSCs. In addition, a colony formation assay was used to examine whether silencing of NF- $\mathrm{KB}$ p65 affected the anti-radioresistance effect of 2-ME2 on NPCSCs. The data showed that 2-ME2 reduced the radioresistance of NPCSCs and that NF- $\mathrm{KB}$ p65 further enhanced the antiradioresistance effects of 2-ME2 (Fig. 4D).

$N F-\kappa B$ p65 knockdown suppresses HIF-1 $\alpha$ mRNA and protein expression and reverses EMT in NPCSCs. RT-PCR and western blot analyses were used to explore the molecular mechanism involved in the effect of NF- $\mathrm{KB}$ p65 on NPCSCs. After NF- $\kappa B$ p65 knockdown, the mRNA and protein levels of p65 and HIF- $1 \alpha$ were significantly decreased (Fig. 5A). The protein levels of the EMT markers $\mathrm{N}$-cad and vimentin were decreased, whereas the protein level of E-cad was increased (Fig. 5B). These results suggested that NF- $\kappa$ B p65 knockdown inhibited HIF-1 $\alpha$ and reversed EMT in NPCSCs.

\section{Discussion}

Previous studies have confirmed that CSCs can be collected quickly and efficiently in a serum-free culture system $(25,26)$. Therefore, a serum-free culture system was used in this study. According to these stemness identification results, compared with the parental cells, the stem-like spherical cells presented suspended growth, limitless self-renewal and re-differentiation abilities, overexpression of stem-relevant genes, a higher proportion of $\mathrm{CD}_{133^{+}}$cells, stronger migration ability, and radioresistance. These results indicated that these stem-like spherical cells possess strong stemness, therefore, these cells were used in the subsequently described experiments as NPCSCs.

In the human body, 2-ME2 is an estrogen metabolite. The anticancer effect of 2-ME2 is currently being researched in studies (14). Recent studies have found that 2-ME2 regulates the cell cycle, apoptosis, transcription factor (including NF- $\kappa B, H I F-1)$ activation, and mitosis and that 2-ME2 plays an important role in the therapy resistance of malignant 
prostatic cancer, glioma, and head and neck squamous cell carcinoma $(15-18,23,27,28)$. However, the effect of 2-ME2 on NPCSCs remains unclear. Our study indicates that 2-ME2 inhibits NPCSC growth and migration and reduces NPCSC radioresistance.

$\mathrm{NF}-\kappa \mathrm{B}$ is overexpressed in almost all cancer cells and mediates multiple signaling pathways to contribute to cell proliferation and to treatment resistance (29-31). However, the role of $\mathrm{NF}-\kappa \mathrm{B}$ regulated by $2-\mathrm{ME} 2$ in cancer progression is complex. 2-ME2 displays different effects on $\mathrm{NF}-\kappa \mathrm{B}$ activation in different types of cells. For example, 2-ME2 increased $\mathrm{NF}-\kappa \mathrm{B}$ activity in LNCaP cells, in contrast, 2-ME2 slightly decreased NF- $\mathrm{KB}$ activity in PC3 cells. 2-ME2 can increase NF- $\kappa \mathrm{B}$ activity in tumor cell lines, however, whether this increase stimulates or antagonizes apoptosis appears to be dependent on the specific type of tumor cell (17). 2-ME2 modulation of the downregulation of glucocorticoid receptor (GR) levels is accompanied by $\mathrm{NF}-\kappa \mathrm{B}$ activation in 2-ME2-responsive, but not in 2-ME2-resistant, pancreatic cancer cells (32). 2-ME inhibits the proliferation and induces the apoptosis of leukemia K562 cells via inhibition of NF- $\kappa \mathrm{B}$ activity (15). To determine the mechanism of the anticancer effect of 2-ME2 on NPCSCs, we evaluated the effect of 2-ME2 on NF-кB p65. The western blot analysis and cellular immunofluorescence results revealed that 2-ME2 (0-8 $\mu \mathrm{M})$ reduced the nuclear and cytoplasmic protein expression and nuclear localization of $\mathrm{NF}-\kappa \mathrm{B}$ p 65 at $24 \mathrm{~h}$, which indicated that 2-ME2 inhibited NF- $\kappa \mathrm{B}$ p65 activation in NPCSCs. We also found that HIF-1 $\alpha$ protein expression was decreased in a 2-ME2 concentration-dependent manner. In addition, evidence suggests that 2-ME2 can depolymerize microtubules and inhibit the nuclear accumulation and transcription activity of HIF-1 $\alpha(33,34)$. 2-ME2 has been shown to decrease the activity of HIF-1 $\alpha$ and affect the expression of downstream genes in head and neck squamous cell carcinoma (23). The radiosensitization effects of 2-ME2 may be partially dependent on HIF-1 $\alpha$ inhibition in various tumors (35). Therefore, we speculated that 2-ME2 inhibits the NF- $\kappa \mathrm{B} / \mathrm{HIF}-1$ signaling pathway.

For further study, we knocked down NF- $\mathrm{B}$ p65 using gene interference technology. We found that NF- $\mathrm{B}$ p65 knockdown enhanced the antitumor effect of 2-ME2 on NPCSCs and that the protein and mRNA levels of HIF-1 $\alpha$ decreased along with $\mathrm{NF}-\kappa \mathrm{B}$ p65, which implied that positive regulation of NF- $\kappa \mathrm{B}$ p65 to HIF-1 $\alpha$ existed in NPCSCs, corresponding with previous evidence that $\mathrm{NF}-\kappa \mathrm{B}$ can regulate $\mathrm{HIF}-1 \alpha$ at the mRNA level $(36,37)$. Our previous study determined that NF- $\kappa$ B p 65 knockdown reduced the chemoresistance of NPCSCs via EMT reversal (24). Our subsequent experiments determined that NF- $\mathrm{B}$ p65 knockdown inhibited EMT and reduced the radioresistance of NPCSCs. Based on the above evidence, we conclude that NF- $\mathrm{B}$ p 65 knockdown may enhance the effect of 2-ME2 on NPCSCs via HIF-1 $\alpha$ downregulation and EMT reversal.

Overall, our findings indicate that 2-ME2 inhibits NPCSC proliferation, migration and radioresistance possibly by inhibiting the NF- $\kappa \mathrm{B} / \mathrm{HIF}-1$ signaling pathway and reversing EMT. 2-ME2 has a potential use as a novel tumor therapy drug or radiotherapy sensitization agent, however, additional research and evidence are needed.

\section{Acknowledgements}

This study was supported by the General Program of the National Natural Science Foundation of China (nos. 81171365 and 81560444).

\section{References}

1. Peng G, Wang T, Yang KY, Zhang S, Zhang T, Li Q, Han J and Wu G: A prospective, randomized study comparing outcomes and toxicities of intensity-modulated radiotherapy vs. conventional two-dimensional radiotherapy for the treatment of nasopharyngeal carcinoma. Radiother Oncol 104: 286-293, 2012.

2. Wang R, Wu F, Lu H, Wei B, Feng G, Li G, Liu M, Yan H, Zhu J, Zhang Y, et al: Definitive intensity-modulated radiation therapy for nasopharyngeal carcinoma: Long-term outcome of a multicenter prospective study. J Cancer Res Clin Oncol 139: 139-145, 2013.

3. Yang CF, Peng LX, Huang TJ, Yang GD, Chu QQ, Liang YY, Cao X, Xie P, Zheng LS, Huang HB, et al: Cancer stem-like cell characteristics induced by EB virus-encoded LMP1 contribute to radioresistance in nasopharyngeal carcinoma by suppressing the p53-mediated apoptosis pathway. Cancer Lett 344: 260-271, 2014.

4. Wang WJ, Wu SP, Liu JB, Shi YS, Huang X, Zhang QB and Yao KT: MYC regulation of $\mathrm{CHK} 1$ and $\mathrm{CHK} 2$ promotes radioresistance in a stem cell-like population of nasopharyngeal carcinoma cells. Cancer Res 73: 1219-1231, 2013.

5. Wei P, Niu M, Pan S, Zhou Y, Shuai C, Wang J, Peng S and Li G: Cancer stem-like cell: A novel target for nasopharyngeal carcinoma therapy. Stem Cell Res Ther 5: 44, 2014.

6. Zhu Y, Liu H, Xu L, An H, Liu W, Liu Y, Lin Z and Xu J: p21-activated kinase 1 determines stem-like phenotype and sunitinib resistance via NF- $\kappa \mathrm{B} / \mathrm{IL}-6$ activation in renal cell carcinoma. Cell Death Dis 6: e1637,2015.

7. Vinogradov S and Wei X: Cancer stem cells and drug resistance: The potential of nanomedicine. Nanomedicine (Lond) 7 : 597-615, 2012.

8. Meijer TW, Kaanders JH, Span PN and Bussink J: Targeting hypoxia, HIF-1, and tumor glucose metabolism to improve radiotherapy efficacy. Clin Cancer Res 18: 5585-5594, 2012.

9. Scholz CC, von Kriegsheim A, Tambuwala MM, Hams E, Cheong A, Bruning U, Fallon PG, Cummins EP and Taylor CT: Prolyl Hydroxylase 1 (PHD1) and Factor Inhibiting HIF (FIH) regulate IL-1 $\beta$-induced NF- $\mathrm{BB}$ activity linking key hypoxic and inflammatory signaling pathways. FASEB J 27: 717-719, 2013.

10. Wong JH, Lui VW, Umezawa K, Ho Y, Wong EY, Ng MH, Cheng SH, Tsang CM, Tsao SW and Chan AT: A small molecule inhibitor of NF-kappaB, dehydroxymethylepoxyquinomicin (DHMEQ), suppresses growth and invasion of nasopharyngeal carcinoma (NPC) cells. Cancer Lett 287: 23-32, 2010.

11. Kan R, Shuen WH, Lung HL, Cheung AK, Dai W, Kwong DL, Ng WT, Lee AW, Yau CC, Ngan RK, et al: NF- $\kappa$ B p65 subunit is modulated by Latent Transforming Growth Factor- $\beta$ Binding Protein 2 (LTBP2) in nasopharyngeal carcinoma HONE1 and HK1 cells. PLoS One 10: e0127239, 2015.

12. Li CW, Xia W, Huo L, Lim SO, Wu Y, Hsu JL, Chao CH, Yamaguchi H, Yang NK, Ding Q, et al: Epithelial-mesenchymal transition induced by TNF- $\alpha$ requires NF- $\mathrm{KB}$-mediated transcriptional upregulation of Twist1. Cancer Res 72: 1290-1300, 2012.

13. Zhao D, Tang XF, Yang K, Liu JY and Ma XR: Over-expression of integrin-linked kinase correlates with aberrant expression of Snail, E-cadherin and N-cadherin in oral squamous cell carcinoma: Implications in tumor progression and metastasis. Clin Exp Metastasis 29: 957-969, 2012.

14. Kambhampati S, Rajewski RA, Tanol M, Haque I, Das A, Banerjee S, Jha S, Burns D, Borrego-Diaz E, Van Veldhuizen PJ, et al: A second-generation 2-Methoxyestradiol prodrug is effective against Barrett's adenocarcinoma in a mouse xenograft model. Mol Cancer Ther 12: 255-263, 2013.

15. Zhang XY,Zhan R, Huang HB and Yang T: Mechanism underlying 2-methoxyestradiol inducing apoptosis of K562 cells. Zhongguo Shi Yan Xue Ye Xue Za Zhi 17: 340-344, 2009 (In Chinese).

16. Kumar AP, Garcia GE, Orsborn J, Levin VA and Slaga TJ: 2-Methoxyestradiol interferes with NFKB transcriptional activity in primitive neuroectodermal brain tumors: Implications for management. Carcinogenesis 24: 209-216, 2003. 
17. Parrondo R, de las Pozas A, Reiner T, Rai P and Perez-Stable C: $\mathrm{NF}-\kappa \mathrm{B}$ activation enhances cell death by antimitotic drugs in human prostate cancer cells. Mol Cancer 9: 182, 2010.

18. Semenza GL: Hypoxia-inducible factors: Mediators of cancer progression and targets for cancer therapy. Trends Pharmacol Sci 33: 207-214, 2012.

19. Marie-Egyptienne DT, Lohse I and Hill RP: Cancer stem cells, the epithelial to mesenchymal transition (EMT) and radioresistance: Potential role of hypoxia. Cancer Lett 341: 63-72, 2013

20. Conley SJ, Baker TL, Burnet JP, Thiesen RL, Lazarus D, Peters CG, Clouthier SG, Elias of S and Wicha MS: CRLX101, an investigational camptothecin-containing nanoparticle-drug conjugate, reverses the HIF- $1 \alpha$-mediated increase in cancer stem cells caused by bevacizumab in a preclinical model of triple-negative breast cancer. Cancer Res 75 (Suppl 15): 1384, 2015.

21. Kazi AA, Shah P, Schech A, Sabnis G, Chumsri S and Brodie A Inhibition of non-hypoxic HIF-1 expression in letrozole-resistant breast cancer cells reduces their cancer stem cell characteristics. Cancer Res 73 (Suppl 8): 95, 2013.

22. Gammon L and Mackenzie IC: Roles of hypoxia, stem cells and epithelial-mesenchymal transition in the spread and treatment resistance of head and neck cancer. J Oral Pathol Med 45: 77-82, 2016

23. Ricker JL, Chen Z, Yang XP, Pribluda VS, Swartz GM and Van Waes C: 2-methoxyestradiol inhibits hypoxia-inducible factor $1 \alpha$, tumor growth, and angiogenesis and augments paclitaxel efficacy in head and neck squamous cell carcinoma. Clin Cancer Res 10: 8665-8673, 2004.

24. Li YJ, Wu SL, Lu SM, Chen F, Guo Y, Gan SM, Shi YL, Liu S and Li SL: (-)-Epigallocatechin-3-gallate inhibits nasopharyngeal cancer stem cell self-renewal and migration and reverses the epithelial-mesenchymal transition via NF- $\mathrm{B}$ p 65 inactivation. Tumour Biol 36: 2747-2761, 2015.

25. Fan X, Liu S, Su F, Pan Q and Lin T: Effective enrichment of prostate cancer stem cells from spheres in a suspension culture system. Urol Oncol 30: 314-318, 2012.

26. Chen SF, Chang YC, Nieh S, Liu CL, Yang CY and Lin YS: Nonadhesive culture system as a model of rapid sphere formation with cancer stem cell properties. PLoS One 7: e31864, 2012.

27. Kang SH, Cho HT, Devi S, Zhang Z, Escuin D, Liang Z, Mao H, Brat DJ, Olson JJ, Simons JW, et al: Antitumor effect of 2-methoxyestradiol in a rat orthotopic brain tumor model. Cancer Res 66: 11991-11997, 2006.
28. Chua YS, Chua YL and Hagen T: Structure activity analysis of 2-methoxyestradiol analogues reveals targeting of microtubules as the major mechanism of antiproliferative and proapoptotic activity. Mol Cancer Ther 9: 224-235, 2010.

29. Maier HJ, Schmidt-Strassburger U, Huber MA, Wiedemann EM, Beug $\mathrm{H}$ and Wirth T: NF-кB promotes epithelial-mesenchymal transition, migration and invasion of pancreatic carcinoma cells. Cancer Lett 295: 214-228, 2010.

30. Liu M, Sakamaki T, Casimiro MC, Willmarth NE, Quong AA, Ju X, Ojeifo J, Jiao X, Yeow WS, Katiyar S, et al: The canonical $\mathrm{NF}-\kappa \mathrm{B}$ pathway governs mammary tumorigenesis in transgenic mice and tumor stem cell expansion. Cancer Res 70: 10464-10473, 2010.

31. Lin Y, Bai L, Chen $\mathrm{W}$ and Xu S: The NF- $\mathrm{B}$ activation pathways, emerging molecular targets for cancer prevention and therapy. Expert Opin Ther Targets 14: 45-55, 2010.

32. Ray G, Dhar G, Van Veldhuizen PJ, Banerjee S, Saxena NK, Sengupta K and Banerjee SK: Modulation of cell-cycle regulatory signaling network by 2 -methoxyestradiol in prostate cancer cells is mediated through multiple signal transduction pathways. Biochemistry 45: 3703-3713, 2006.

33. Siebert AE, Sanchez AL, Dinda S and Moudgil VK: Effects of estrogen metabolite 2-methoxyestradiol on tumor suppressor protein p53 and proliferation of breast cancer cells. Syst Biol Reprod Med 57: 279-287, 2011.

34. Pasquier E, André N and Braguer D: Targeting microtubules to inhibit angiogenesis and disrupt tumour vasculature: Implications for cancer treatment. Curr Cancer Drug Targets 7: 566-581, 2007.

35. Moeller BJ, Cao Y, Li CY and Dewhirst MW: Radiation activates HIF-1 to regulate vascular radiosensitivity in tumors: Role of reoxygenation, free radicals, and stress granules. Cancer Cell 5: 429-441, 2004

36. Walmsley SR, Print C, Farahi N, Peyssonnaux C, Johnson RS, Cramer T, Sobolewski A, Condliffe AM, Cowburn AS, Johnson $\mathrm{N}$, et al: Hypoxia-induced neutrophil survival is mediated by HIF-1 $\alpha$-dependent NF-кB activity. J Exp Med 201: 105-115, 2005.

37. Taylor CT: Interdependent roles for hypoxia inducible factor and nuclear factor- $\mathrm{\kappa B}$ in hypoxic inflammation. J Physiol 586: 4055-4059, 2008. 